\title{
Variations
}

Variations

Revue internationale de théorie critique

$24 \mid 2021$

Echos

\section{Le fétiche travail et son empire : la critique de l'économie comme critique de la forme valeur?}

Jean-Marie Vincent

\section{(2) OpenEdition}

Journals

Édition électronique

URL : https://journals.openedition.org/variations/2043

DOI : 10.4000/variations.2043

ISSN : 1968-3960

Éditeur

Les amis de Variations

Référence électronique

Jean-Marie Vincent, «Le fétiche travail et son empire : la critique de l'économie comme critique de la forme valeur? », Variations [En ligne], 24 | 2021, mis en ligne le 03 juillet 2021, consulté le 07 juillet 2021. URL : http://journals.openedition.org/variations/2043 ; DOI : https://doi.org/10.4000/variations. 2043

Ce document a été généré automatiquement le 7 juillet 2021.

Les ami•e•s de Variations 


\title{
Le fétiche travail et son empire : la critique de l'économie comme critique de la forme valeur?
}

\author{
Jean-Marie Vincent
}

\section{NOTE DE L'ÉDITEUR}

Ce texte est extrait de l'ouvrage de Jean-Marie Vincent, Critique du travail. Le faire et l'agir publié pour la première fois au PUF en 1987, réédité aux éditions critiques en 2019. Une version numérique complète est disponible en ligne sur le site de Variations, avec l'aimable autorisation des ayants-droit.

Le livre se situe à la croisée des chemins, entre la fin d'un monde et la quête d'un nouveau souffle critique. La gauche est alors en train de péricliter. Le gouvernement socialiste vient d'organiser le " tournant de la rigueur » sous Mitterrand, tandis que le communisme stalinien livre une dernière parade triste avant la chute du mur de Berlin. Sur le plan intellectuel, nombre d'anciens marxistes épousent le tournant culturaliste qui évacue la critique du capitalisme de la scène théorique (Alain Touraine), quand ils ne se livrent pas à des confusions idéologiques sous l'égide des nouveaux philosophes, qui ont fini en vieux sarkozystes (André Glucksmann, Alain Finkielkraut). Tout devait disparaitre, les utopies, le prolétariat, la critique et même l'art contemporain, emportés par la mort des grands récits. Le livre ne donna lieu à aucun un baroud d'honneur mais inaugura plutôt un espace de discussion aux côtés de Toni Negri, André Gorz, Michael Löwy, de féministes comme Jacqueline Heinen et d'autres universitaires attachés à l'esprit de l'Université de Vincennes. Le département de sciences politiques, que dirigeait alors Jean-Marie Vincent, vit ainsi naître la revue Futur antérieur qui, sous sa direction, anima les débats de la gauche critique jusqu'en 1999, date de l'essor du mouvement altermondialiste à Seattle. Puis Vincent prolongea avec Variations l'expérience d'un Futur antérieur qui engendre aujourd'hui encore quelques légendes urbaines. 
1 Pour Marx, il s'agit d'un monde renversé, la tête (en l'occurrence, les hommes agissant) en bas, dans lequel les rapports sociaux, séparés de leurs supports vivants, ont lieu entre les choses, ces choses particulières que sont les marchandises. Le mort l'emporte sur le vif, le suprasensible sur le sensible : la dialectique est en quelque sorte le résultat d'un renversement qui met toute la société sens dessus dessous. Mais qu'on y prête bien attention, Marx n'affirme pas que cette inversion est perversion, qu'on peut l'analyser en simples termes de déperdition, de perte de contenu, il essaye de montrer qu'elle est contraire ou opposée à un certain nombre de processus (entre autres le métabolisme homme nature, les communications humaines et les échanges symboliques, etc.), dont la dialectique de la forme valeur ne peut jamais totalement se séparer ou s'affranchir. Une dualité irréductible marque fondamentalement la société capitaliste, mais il serait erroné d'y voir la scission d'une unité originaire et le résultat d'une histoire continue et homogène dans ses composantes essentielles. La dualité doit plutôt être interprétée comme une duplicité, comme une incompatibilité latente et récurrente entre l'idéalisme de la valeur et du Capital (le dépassement permanent des limites, la négation idéale des obstacles matériels) et la rationalité (la détermination finie) des interactions concrètes entre les hommes et des échanges avec le monde. La société capitaliste peut ainsi être comprise comme le lieu de déséquilibres et de désordres incessants sans que pour autant le sens de son dépassement soit donné une fois pour toutes, car il dépend au fond des déplacements qui se produisent entre monde sensible suprasensible et monde sensible. C'est dire l'importance de leur articulation, des formes de leur jonction et de leur disjonction que Marx n'aborde jamais de façon systématique, mais de façon tout de même relativement explicite tant dans les Manuscrits de 1861/1863 que dans Le Capital, notamment en traitant des problèmes de la circulation et de la superficie phénoménale de la société. Comme il le remarque dans le chapitre II « Des échanges » du livre I du Capital, pour que les marchandises entrent en rapport les unes avec les autres, il faut que les volontés de leurs conducteurs ou possesseurs les habitent en tant que choses et se rencontrent pour aboutir à des actes volontaires communs. Les mouvements de la valeur passent en ce sens par les échanges de volonté subjectifs des représentants des marchandises et «l'objectivité » du monde de la marchandise trouve sa présupposition dans le subjectivisme d'échangistes qui ne se préoccupent que de leurs propres prestations. Cela revient à dire que l'extrême d'une objectivité supra-humaine, prenant les apparences de la valeur comme substance sujet, trouve ses conditions de possibilité dans les subjectivités monadiques de volontés qui n'ont plus de points d'application privilégiés, mais se veulent elles-mêmes pour se valoriser par rapport aux autres volontés. La dévalorisation du sensible et de la matérialité sociale au niveau de l'universalité des échanges de marchandises trouve son pendant et son répondant au niveau des individus dans l'ascèse particulière qui leur fait nier de façon récurrente le caractère sensible-matériel de leurs relations et connexions pour se retrouver dans le monde de la valeur. Toutes proportions gardées, l'insertion de l'individu dans l'abstraction de la valeur est analogue à l'odyssée de la conscience telle que Hegel la décrit dans la Phénoménologie de l'esprit: dépassements successifs des stades de la certitude sensible, de la perception, de l'entendement pour arriver au moment où tout bascule, où le monde suprasensible de la loi et de la mise en ordre du multiple cède la place au deuxième monde suprasensible, celui de la conscience qui se sait elle-même dans son être-autre, qui est égale à elle-même dans cet être-autre d'étalon de mesure naturel (à la limite aussi concret que le travail concret). Les grandeurs de valeur ne sont plus secondes, c'est-à-dire déterminées par autre chose 
qu'elles-mêmes, elles deviennent premières, c'est-à-dire déterminantes. C'est à partir de ces glissements théoriques et de ces méprises que Marx se fourvoie, sans pouvoir en sortir, dans l'impasse du problème dit de la transformation des valeurs en prix de production. Il donne au problème une solution qui n'en est pas une (en faisant abstraction de la nécessaire transformation de la valeur des capitaux en prix de production), et croit pouvoir égaliser masse globale de la plus-value et masse globale du profit à partir de ces prémisses. Il lui est de cette façon impossible de saisir que les prix de production ne dérivent pas des grandeurs de valeur, mais des conditions de production (effectivité des techniques de production) et des variations contraires ou opposées du profit et des salaires. Il ne peut par suite renvoyer les grandeurs de valeur (l'homogénéisation et la quantification du travail abstrait) à leur véritable origine, la polarité des rapports de classe et la productivité physique du travail. Les grandeurs de valeur ne sont pas prises pour ce qu'elles sont, c'est-à- dire des variables dépendantes (au-delà du temps de travail socialement nécessaire et de l'égalisation abstraite de travaux multiples au niveau de la production et de l'échange), des rapports de force cristallisés entre les classes et des rapports technico-cognitifs aux moyens de production impliqués dans ces rapports de classe. Les grandeurs de valeur sont en quelque sorte substantifiées, dans l'oubli de leur nature relationnelle, comme si elles étaient l'expression quantitative d'une substance « naturelle » temps de travail.

2 Engagé sur cette voix, Marx se trouve involontairement, mais inévitablement déporté vers une conception quelque peu «naturaliste» des contradictions économiques du capitalisme. Sans jamais disparaître complètement, les problèmes de compatibilité et d'incompatibilité entre les niveaux dialectiques et sensibles de la réalité sociale sont peu à peu relégués au second plan, et cela au profit des oppositions ou des contradictions jouant pour l'essentiel au niveau de l'accumulation. Le mouvement de la valorisation tend par là à être analysé comme produisant lui-même et de façon linéaire ses propres limites; les contradictions économiques s'engendrant les unes les autres selon une dynamique qui accentue les difficultés du capitalisme. Un bon exemple de cette orientation, jamais vraiment explicitée, est donné par la loi de la baisse tendancielle du taux de profit (et son présupposé l'élévation de la composition organique du Capital).

3 En la formulant, Marx essaye, à l'évidence, d'approcher au plus près le mouvement d'ensemble ou la logique de l'accumulation du capital en évitant les prévisions trop mécanistes ou déterministes. Dans la mesure où il reste toutefois étroitement centré sur le niveau économique et sur les variations des grandeurs de valeur, il lui échappe que les tendances à la baisse du taux de profit ne sont pas seulement contrebalancées ou temporairement mises hors-jeu par des contre-tendances, mais qu'elles peuvent être complètement invalidées à un moment donné par l'établissement de nouveaux rapports entre la valorisation et le matériel sensible. Comme le disent dorénavant beaucoup d'économistes marxistes, il n'y a pas sur longue période de loi d'évolution du profit à la baisse, et on ne peut pas non plus y trouver l'élément fondamental d'explication des crises économiques (il faut à ce propos chercher des théorisations complexes et pluralistes, sur ces problèmes la littérature est immense).

4 Aussi faut-il bien constater que Marx, malgré sa volonté de contribuer à une économie politique en acte de la classe ouvrière, n'arrive pas à formuler clairement le projet d'une économie politique de la réversion de l'inversion, c'est-à-dire une discipline qui restitue l'ensemble des mouvements matériels-sensibles qui supportent la valorisation, 
en démontant en même temps les mécanismes de représentation sociale qui fonctionnent comme des mécanismes de rigidification du sens, de détournement de significations par substitution des unes aux autres (certains mouvements et relations devenant les manifestations de ce qu'ils ne sont pas et ne peuvent être). En bref, dans les énoncés marxiens, la critique de l'économie politique n'apparaît pas encore comme un déchiffrement de flux matériels et immatériels entrelacés qui puisse conduire à une mise en question radicale des dispositifs les plus fondamentaux (cognitifs, praticoéthiques, pratico-techniques, sensuels-libidinaux, etc.), des rapports des hommes entre eux et au monde. La critique de l'économie politique, au-delà de ses points d'application immédiats, se doit pourtant d'être critique des formes sclérosées de l'intersubjectivité (communications déviées par la valorisation) qui, en faisant des individus des sujets-Robinsons, les rendent incapables de penser leurs liens aux autres, à l'action et à la matérialité autrement qu'en termes de volonté et de domination. Elle ne peut pas ne pas être destruction de l'ontologie sous-jacente au monde des valeurs (spirituelles comme matérielles) et de la volonté, non par une quelconque prédilection rétrospective pour les univers hiérarchisés antérieurs au polythéisme des valeurs, mais pour reposer de façon radicalement différente les relationnalités hommes nature, voire pour instaurer de nouveaux rapports entre l'action et l'imaginaire social. En suivant cette orientation, elle devrait, entre autres, montrer que la technique (y compris sous la forme d'élaborations scientifiques) n'est pas à la disposition des hommes, mais se montre tout à fait soumise à la logique du travail abstrait comme rapport social à reproduire et comme rapport social à l'objectivité et à la subjectivité. La technique n'est en effet pas pure instrumentalité (adéquation des moyens aux fins), elle véhicule des représentations et des conceptions du monde qui prédéterminent la façon d'aborder et de connaître les processus et les échanges matériels. Elle n'est pas par elle-même domination ou oppression, mais elle transmet dans son " objectivité » la plus détachée (respecter les processus extra-humains pour mieux les canaliser) de fortes charges de subjectivité unilatérale qui nient ou occultent une partie des relations au monde. Il ne peut donc être question de réduire les questions qu'elle soulève à celles qui sont inhérentes à l'emploi capitaliste des machines comme Marx semble le laisser entendre dans Le Capital.

Elle pose plus fondamentalement le problème de la nature et de la forme des forces productives, en tant qu'imbrication de forces productives humaines et matérielles. A ce propos, les textes de Marx ouvrent effectivement des perspectives intéressantes, notamment celle de forces productives humaines libérées, c'est-à-dire affranchies des contraintes de la mise en valeur du capital, mais sur ce point aussi ils donnent l'impression de ne pas aller assez loin dans le questionnement. On peut se demander en particulier si les difficultés rencontrées pour maitriser les processus technologiques et reconstruire sur d'autres bases la symbiose hommes-machines ne sont pas sousestimées ou ramenées à des considérations trop étroitement afférentes à la production matérielle et à la production en général. La libération des forces productives humaines qui est forcément libération par rapport au travail abstrait et à sa domination envahissante a, par suite, une dimension extra-productive qu'il peut être dangereux de vouloir ignorer.

6 Aussi est-ce seulement à condition de se soustraire à une dialectique sujet-objet réductrice que l'imaginaire social peut faire naître de nouvelles façons de pratiquer la société et le monde tout en permettant d'utiliser des systèmes de machines et des 
agencements systémiques (automatismes sociaux) comme prolongement véritable des actions humaines.

7 Les imprécisions, les hésitations et les incertitudes dans la critique marxienne de l'économie politique ont évidemment obscurci ou masqué l'audace de son dessein et le caractère novateur de beaucoup de ses aperçus. Après la mort de Marx la discipline critique de l'économie devient pour l'essentiel une variante de l'économie politique dont la préoccupation première est de formuler les lois d'évolution du capitalisme. Cela se manifeste d'abord par une acceptation a-critique d'une théorie « naturaliste » de la valeur qui doit plus à Ricardo qu'à Marx. Les marxistes, il est vrai, prennent leur distance par rapport aux socialistes ricardiens qui proclament le droit au produit intégral du travail et considèrent l'exploitation comme une atteinte à l'intégrité physique et morale du travailleur. Mais, à y regarder de plus près, les disciples de Marx ne s'éloignent pas tellement de la thématique ricardienne lorsqu'ils font du travail une sorte d'élément premier - supra-historique - de l'organisation sociale. Le travail abstrait n'est pas conçu par eux comme une substance-sujet produite par des relations et des représentations sociales, mais bien comme une substance commune à tous les produits de l'activité productrice humaine, au-delà des différences de sociétés. Le socialisme comme maitrise collective des processus de travail et comme disposition démocratiquement décidée du surtravail est ainsi révélation du travail à lui-même et révélation de la société à elle-même. La société socialiste met le travail à sa vraie place et le dépérissement de la loi de la valeur se fait jour comme disparition progressive des mécanismes de marché (marché des biens de production et marché du travail) et progression de l'allocation planifiée du travail social direct entre les différentes branches de production. C'est bien pourquoi on peut critiquer l'économie capitaliste comme une économie de l'utilisation irrationnelle du travail (gaspillage, chômage, etc.), et de l'anarchie a-sociale au niveau économique. Les contradictions économiques, insuffisance de la consommation solvable par rapport à la production, disproportions de l'accumulation entre les différentes branches de l'économie, baisse du taux de profit, suraccumulation, etc., sont là pour démontrer que le capitalisme comporte des vices de fonctionnement irrémédiables. Sans doute, les esprits se séparent-ils sur les issues possibles, écroulement du système sous le poids de ses contradictions, prise de conscience des plus larges masses devant les effets négatifs de la perpétuation des rapports capitalistes, transformation progressive des mécanismes socio-économiques pour faire face à des difficultés récurrentes. Cela n'empêche pourtant pas l'immense majorité des marxistes de croire que la fin du capitalisme est, sinon programmée dans le détail, du moins inscrite dans ses caractéristiques les plus fondamentales en tant que système économique. La théorie critique du capitalisme n'a, en ce sens, pas besoin d'aller au-delà d'une théorie du déclin ou de la décadence des relations capitalistes de production. Ce qui, en clair, veut dire qu'elle n'a pas à être élucidation de ses conditions de développement et de reproduction élargie. Encore moins doit-elle se préoccuper des mutations qui peuvent se produire dans les relations sociales, dans les champs d'action offerts aux individus et plus généralement dans l'horizon des pratiques sociales. Faire la critique du capitalisme, ce n'est pas déplacer sans cesse les problématiques théoriques, découvrir ce qui se dérobe sous les apparences de la continuité ou être attentif aux failles qui se creusent dans le bel ordonnancement des rapports sociaux, c'est seulement enregistrer ce qui se passe sous le regard pour y trouver les raisons d'un avenir abstraitement autre. 
Il est au fond très significatif que la critique de l'économie politique soit devenue chez la plupart des marxistes une théorie pleinement positiviste de l'économie, de ses mouvements nécessaires, de son évolution et de ses cycles selon des lignes prédéterminées, c'est-à-dire une discipline présentant beaucoup d'analogies avec son adversaire, l'économie politique "bourgeoise ». Chez les uns et chez les autres, il y a le même attachement à un modèle étroit de science sociale et à la méthodologie qui en découle. Sans doute, les prémisses ou les références de base tendancielles à différer beaucoup après l'adoption par la plupart des économistes «bourgeois » de théories «subjectives » de la valeur, ce qui explique que de nombreux marxistes puissent croire de bonne foi qu'ils font œuvre critique en opposant leur conception «objective» de la valeur au subjectivisme des marginalistes. Mais cela ne doit pas cacher le fait que les antagonistes ne font que s'opposer sur les substrats «naturels » de la valeur, travail ou utilité, c'est-à-dire sur la façon de juger les activités qui se confrontent dans la circulation et la production, travail de supervision et de surveillance d'un côté; travail salarié d'exécution de l'autre. Dans un cas, le recours à la psychologie rationaliste de l'utilité marginale et de la combinaison optimale des facteurs de production reflète et justifie les rôles capitalistes (accumulation, innovation, etc.), dans l'autre cas l'affirmation du rôle primordial du travail dépendant dans la production des richesses exprime au niveau théorique les efforts d'auto-appréciation des salariés dans leur résistance à l'exploitation. Il s'agit bien de deux « subjectivités » sociales qui cherchent à trouver des fondements «objectifs» à leurs pratiques; les points de vue divergent indéniablement, mais ils n'empêchent pas les économistes des deux camps de se rejoindre dans la fétichisation de l'économie, de cette économie qu'ils partagent, même en s'affrontant durement. On peut être tenté, il est vrai, de récuser cette analyse en rappelant l'historicisme résolu des marxistes qui soulignent presque tous l'inachèvement ou l'imperfection du capitalisme comme mode de production. Mais n'y a-t-il pas là un historicisme naturalisé ou un naturalisme historicisé qui prétend élucider une dynamique sociale à partir de quelques matrices de base, aisément repérables et identifiables? Il serait, certes, injuste de réduire les marxistes à un naturalisme borné dans leurs meilleurs développements et intuitions, mais force est d'admettre que les percées au-delà de la théorie ricardienne de la valeur-travail sont rares et irrégulières, et ne sont jamais sérieusement exploitées. Aussi bien la critique de l'économie politique est-elle encore aujourd'hui une discipline en filigrane, plus potentielle que véritablement constituée en tant que discours articulé et systématique. A partir des premières indications données par Marx, il reste toujours à mettre au point ses grandes lignes de force, ses modalités d'attaque des deux niveaux du réel social (dialectique et non dialectique). Il lui faut notamment saisir les mouvements de l'économie dans leur duplicité, dans la série de dédoublements successifs qui les caractérisent au niveau des relations comme des pratiques. Mais, pour aller plus au fond, il lui faut surtout comprendre l'interaction des deux mondes, leur façon de s'influencer et de se conditionner réciproquement dans un jeu apparemment sans terme, bien que dominé par la dialectique des formes sociales objectives.

On est en particulier obligé de dépasser les oppositions simples entre le monde « ensorcelé » de la valeur et de la marchandise d'un côté, le monde « authentique » des métabolismes matériels et sensibles de l'autre. Chacun des deux pénètre l'autre, se représente et s'affirme grâce à l'autre. En ce sens, les abstractions réelles ont pour fonction de faire passer d'un niveau de réalité à l'autre avant même que d'être des barrières ou des obstacles à une perception globale des processus. Les flux de la 
production et des échanges en tant que combinaison matérielle sensible de transformations et de translations trouvent direction et sens dans les codes et les signes qui informent les relations marchandes entre équivalents. De même les rapports dialectiques entre les formes de la valeur se nourrissent de déplacements et de mutations matériels mis en œuvre par les hommes, tout cela en vue de s'affirmer contre eux. Il s'opère en permanence des permutations et des substitutions qui transfigurent la scène sociale en jeux en trompe-l'œil, pleins de quiproquos et de télescopages, où la réalité se dérobe comme toujours autre et toujours double. La valeur d'usage est une condition de possibilité de la valeur d'échange, mais elle-même ne peut être produite que grâce au mouvement des valeurs d'échange. Le travail concret porte le travail abstrait, mais c'est la répartition de ce dernier entre les différentes branches de la production et de la circulation qui dicte au travail concret ses caractéristiques concrètes. Ces relations d'inséparabilité ne s'épuisent pas toutefois les unes dans les autres. Les codes et les signes de la valeur qui se valorise ne peuvent dire toutes les significations attachées aux flux matériels et immatériels des pratiques sociales et ces dernières ne se coulent qu'imparfaitement dans les moules des formes sociales. Les abstractions réelles en tant que points de passage ou de communication entre ces mondes homogènes/hétérogènes ne peuvent convertir parfaitement, terme à terme, le matériel-sensible en sensible- suprasensible, et vice versa.

Il subsiste toujours, du côté dialectique comme du côté non dialectique, des excédents difficilement assimilables, une plus-value de sens du matériel-sensible comme des valeurs qui ne peuvent se valoriser dans la sphère du suprasensible. Il en résulte des séries quasi ininterrompues de pressions et de contrepressions d'un monde sur l'autre. Les forces d'attraction semblent engendrer dans leur mouvement même de vigoureuses forces de répulsion dans un climat général de reproduction instable. Les processus d'intégration l'un dans l'autre des niveaux du réel sont concomitants de tendances au rejet et à la désintégration qui marquent la plupart des manifestations sociales du sceau de l'ambiguité et de la tension avec soi-même.

11 Cela est particulièrement vrai de la lutte des classes qui, chez les travailleurs, est à la fois résistance aux impératifs de la mise en valeur du capital, et lutte pour la valorisation de la force de travail contre les différentes formes de sa dévalorisation. Elle peut aussi bien entraver l'accumulation du capital que la stimuler de façon remarquable. Elle est, par conséquent, susceptible de véhiculer des significations, sinon diamétralement opposées, du moins non superposables, ce qui ne peut pas ne pas avoir de répercussions sur les modes de fonctionnement de la société. Pour les acteurs individuels et collectifs, les significations des pratiques, des relations et des institutions sociales ne sont jamais définitivement données, et, à partir des abstractions réelles, s'élèvent des abstractions secondaires qui tentent, au prix d'une très rapide usure, de mettre de l'unité dans l'ondoyant et le divers, de l'harmonie dans le conflit. Dans les agencements systémiques autour des abstractions réelles, l'univocité et la permanence du sens ne sont pas en fait assurées une fois pour toutes : la société se fait et se défait selon des séquences discontinues. On peut trouver çà et là des isolats sociaux où la valeur n'a qu'un poids limité (les relations de parenté par exemple), et il n'est pas rare que certaines formes de sociabilité (l'amitié, la camaraderie, la sexualité, etc.) soient au moins partiellement contraires aux exigences de la reproduction. Aussi bien est-il indispensable que la primauté du monde dialectique soit réaffirmée matériellement et idéellement par les institutions de niveau sociétal, par-dessus le bouillonnement et les incongruences dans la confrontation du suprasensible et du sensible. L'État, en 
particulier, doit mettre au point des mécanismes complexes de compensation des défaillances (multiformes) de la valorisation, au besoin en contredisant la logique immédiate de celle-ci (correction des mécanismes de marché, intervention publique dans l'économie, protection sociale, etc.). Comme l'a très bien montré l'école dite de la régulation, le rapport capital-travail ne peut jamais être statique, mais doit au contraire se perpétuer par des réorganisations successives du rapport salarial (mode d'insertion des travailleurs dans la production, la répartition et la consommation). La réglementation juridique elle-même ne peut en rester à la seule régulation des rapports d'échange, elle se doit peu à peu de déborder sur le domaine de la conflictualité sociale et de participer à sa pacification relative.

\section{AUTEUR}

\section{JEAN-MARIE VINCENT}

Directeur du département de Sciences politiques de l'Université Paris 8 (1972-2003). Fondateur de la revue Variations. 